\title{
Author Correction: Sex-Specific Developmental Effects of Father Absence on Casual Sexual Behavior and Life History Strategy
}

\author{
Jessica A. Hehman ${ }^{1}$. Catherine A. Salmon ${ }^{1}$
}

Published online: 22 October 2020

C) Springer Nature Switzerland AG 2020

\section{Author Correction: Evolutionary Psychological Science 5(1):121-130 \\ https://doi.org/10.1007/s40806-018-0173-5}

In the Abstract section, the sentence "When FA occurred during middle childhood, females exhibited faster LH strategies; whereas, when FA occurred during adolescence, males exhibited faster LH strategies." should read as:

"When FA occurred during middle childhood, males exhibited faster LH strategies, whereas when FA occurred during adolescence, females exhibited faster LH strategies."

In the Conclusion section, the sentence "When father absence occurred during middle childhood, females exhibited faster life history strategies whereas males exhibited slower life history strategies. However, when father absence occurred during adolescence, females exhibited slower life history strategies and males exhibited faster life history strategies." should read as:

"When father absence occurred during middle childhood, males exhibited faster life history strategies, whereas females exhibited slower life history strategies. However, when father absence occurred during adolescence, males exhibited slower life history strategies and females exhibited faster life history strategies."

The original article can be found online at https://doi.org/10.1007/ s40806-018-0173-5.

Jessica A. Hehman

jessica_hehman@redlands.edu

1 Psychology Department, University of Redlands, 1200 E.

Colton Ave, Redlands, CA 92373, USA 